\title{
Adipose-Derived Mesenchymal Stem Cells-Derived Exosomes Carry MicroRNA-671 to Alleviate Myocardial Infarction Through Inactivating the TGFBR2/Smad2 Axis
}

\author{
Xue Wang, ${ }^{1}$ Yuhai Zhu, ${ }^{2}$ Chengcheng $W^{1},{ }^{1}$ Wennan Liu, ${ }^{1}$ Yujie He, ${ }^{3}$ and Qing Yang ${ }^{1,4}$
}

(Received February 2, 2021; accepted March 29, 2021)

Abstract-Mesenchymal stem cells (MSCs) and their derived extracellular vesicles have been reported as promising tools for the management of heart disease. The aim of this study was to explore the function of adipose-derived MSCs (adMSCs)-derived exosomes (Exo) in the progression of myocardial infarction (MI) and the molecules involved. Mouse cardiomyocytes were treated with oxygen-glucose deprivation (OGD) to mimic an MI condition in vitro. The adMSCs-derived Exo were identified and administrated into the OGD-treated cardiomyocytes, and then the viability and apoptosis of cells, and the secretion of fibrosis- and inflammation-related cytokines in cells were determined. Differentially expressed microRNAs (miRNAs) in cells after Exo treatment were screened using a microarray analysis. The downstream molecules regulated by miR-671 were explored through bioinformatic analysis. Involvements of miR-671 and transforming growth factor beta receptor 2 (TGFBR2) in the exosome-mediated events were confirmed by rescue experiments. A murine model with MI was induced and treated with Exo for functional experiments in vivo. Compared to phosphate-buffered saline treatment, the Exo treatment significantly enhanced viability while reduced apoptosis of cardiomyocytes, and in reduced myocardial fibrosis and inflammation both in vitro and in vivo. miR-671 was significantly upregulated in cells after Exo treatment. Downregulation of miR-671 blocked the protective functions of Exo. miR-671 targeted TGFBR2 and suppressed phosphorylation of Smad2. Artificial downregulation of TGFBR2 enhanced viability of the OGD-treated cardiomyocytes. This study suggested that adMSC-derived exosomal miR-671 directly targets TGFBR2 and reduces Smad2 phosphorylation to alleviate MI-like symptoms both in vivo and in vitro.

KEY WORDS: myocardial infarction; MSC; exosomes; microRNA-671; TGFBR2; Smad2.

Xue Wang and Yuhai Zhu contributed equally to this work.

${ }^{1}$ Department of Cardiology, Tianjin Medical University General Hospital, NO. 154, Anshan Street, Heping District, Tianjin, 300052, People's Republic of China

${ }^{2}$ Department of Medical Cosmetology, Tianjin Medical University General Hospital Airport Hospital, Tianjin, 300308, People's Republic of China

\footnotetext{
${ }^{3}$ Department of Cardiology, Tianjin Beichen District Chinese Medicine Hospital, Tianjin, 300400, People's Republic of China

${ }^{4}$ To whom correspondence should be addressed at Department of Cardiology, Tianjin Medical University General Hospital, NO. 154, Anshan Street, Heping District, Tianjin, 300052, People's Republic of China. Email: Yangqing9911@163.com
} 


\section{INTRODUCTION}

Acute myocardial infarction (MI) is defined as the acute myocardial injury with the clinical appearance of acute myocardial ischemia along with a rise and/or fall of cardiac troponin values $[1,2]$. MI is commonly known as "heart attack," which induces the formation of noncontractile scar and the remodeling of left ventricular (LV) that further deteriorate cardiac function [3]. The consequential ischemic heart disease is a leading cause of disability and death worldwide [4]. Accumulated LV mass and progressive thickening of the myocardium are typical indicators of LV remodeling and the associated cardiac hypertrophy, apoptosis, and fibrosis [3]. The current clinical treatments for heart failure following MI are instrument implantation and drug use; however, the efficacy remains unsatisfactory [5]. In addition, the surgical interventions and thrombolysis may induce secondary reperfusion injury, leading to further irreversible death of cardiomyocytes [6]. Developing less-harmful and effective alternatives for MI management while limiting cardiomyocyte loss is of great significance.

Upon MI, cardiomyocyte death induces robust inflammatory response which evolves cardiac injury, repair, and remodeling. Exosomes have been increasingly recognized to be implicated in immune regulation and inflammatory response following MI [7]. Exosomes are cell-sourced nanosized vesicles containing molecular substances including microRNAs (miRNAs), lipids, proteins, and nucleic acids and are used as a major type of mediators responsible for intercellular communications in different pathological conditions including ischemic heart disease [8]. Mesenchymal stem cells (MSCs) are one of the most ideal cell therapy tools due to their advantages in easy-isolation, self-renewal, and expansion potentials, and the adipose-derived MSCs (adMSCs) and their released exosomes have seen advantages in clinical application [9]. miRNAs are frequently involved and also play critical functions in exosomemediated events [10]. They are a major class of highly conserved non-coding RNAs involved in a wide array of biological events and cardiac diseases such as MI, heart failure, and cardiomyocyte hypertrophy due to their potent regulation on diverse target genes [11]. Here, our miRNA microarray analysis suggested that miR671 was significantly upregulated in cells after treatment with adMSCs-derived exosomes (hereafter termed Exo; only indicating the exosomes derived from the adMSCs in this study). miR-671 has been reported to be poorly expressed in patients with coronary artery disease [12].
Whether exosomal miR-671 exerts functions in myocardial protection remains unknown. The subsequent integrated analysis suggested transforming growth factor beta (TGF- $\beta$ ) receptor 2 (TGFBR2) as a candidate target gene of miR-671. The TGF- $\beta 1$ signal transduction pathway has been well-recognized to play a crucial role in the process of myocardial fibrosis [13]. Smad proteins, the intracellular effectors of TGF- $\beta$ signaling, are activated by the TGFBRs and translocate into nucleus where they regulate transcription activities [14]. Smad2 and Smad3 are frequently involved in TGF- $\beta 1$-mediated pro-fibrotic events $[15,16]$. TGFBR2 has been reported as an activator of Smad2 phosphorylation [17, 18]. In this study, we hypothesized that Exo contain miR-671 and alleviate MI symptoms through suppression of the TGFBR2/Smad2 axis. Acquired adMSCs were used for Exo extraction, and gain- and loss-of-function studies were performed both in vivo and in vitro to validate the hypothesis.

\section{MATERIALS AND METHODS}

\section{Culture and Identification of Cells}

Mouse adMSCs (CP-M138) and mouse cardiomyocytes (CP-M073) were procured from Procell Life Science \& Technology Co., Ltd. (Wuhan, Hubei, China). The cells were cultured in adMSC-specific medium (CM-M138) and cardiomyocyte-specific medium (CM-M073) in a $37{ }^{\circ} \mathrm{C}$ incubator with $5 \% \mathrm{CO}_{2}$.

To identify the adMSCs, the phenotypic profile of cells was examined by flow cytometry using phycoerythrin-labeled human anti-CD29 (\#102216, Biolegend, San Diego, CA, USA), anti-CD44 (\#338804, Biolegend), anti-CD90 (\#328109, Biolegend), anti-CD45 (\#103106, Biolegend), and anti-immunoglobulin G (IgG, Biolegend). The differentiation potential of adMSCs was examined by osteogenic differentiation (CP1206) and adipogenic differentiation (CP1215) kits (Weitong Biotechnology, Shenzhen, Guangdong, China) using alizarin red staining and Oil red O staining, respectively.

\section{Cell Transfection}

The adMSCs were allocated into adMSC group (without transfection, and the corresponding exosomes were named Exo), NC-inhibitor group (adMSCs were transfected with $\mathrm{NC}$ inhibitor, and the exosomes were named Exo-NC, NC refers to negative control), miR671 inhibitor group (adMSCs were transfected with 
miR-671 inhibitor, and the exosomes were named Exoinhibitor).

The cardiomyocytes were allocated into the following groups after corresponding transfection: Control group, $\mathrm{NC}$ mimic group (cardiomyocytes were transfected with NC mimic), miR-671 mimic (cardiomyocytes were transfected with miR-671 mimic), oxygen-glucose deprivation (OGD) group (cardiomyocytes underwent OGD treatment), phosphate-buffered saline (PBS) group (OGD-induced cardiomyocytes were further treated with PBS for 24 h), Exo group (OGD-induced cardiomyocytes were further treated with Exo for $24 \mathrm{~h}$ ), Exo-NC group (OGD-induced cardiomyocytes were further treated with Exo-NC for $24 \mathrm{~h}$ ), Exo-inhibitor group (OGD-induced cardiomyocytes were further treated with Exo-inhibitor for $24 \mathrm{~h}$ ), Exo-inhibitor + si-NC (small interfering-NC) group (OGD-induced cardiomyocytes were treated with Exo-inhibitor for $24 \mathrm{~h}$ and then transfected with si-NC), and Exo-inhibitor + si-TGFBR2 group (OGD-induced cardiomyocytes were treated with Exo-inhibitor for $24 \mathrm{~h}$ and then transfected with si-TGFBR2). In all cellular experiments involving Exo treatment (except for the Exo uptake experiment), the cardiomyocytes were pre-treated with OGD before Exo treatment. The Exo were administrated after OGD at different time points to determine the suitable interval (see details in the result section).

The dose and duration of Exo administration in cells was $50 \mu \mathrm{g} / \mathrm{mL}$ for $24 \mathrm{~h}$. The miR-671 mimic/inhibitor, siTGFBR2, and the controls were acquired from GenePharma Co., Ltd. (Shanghai, China). All transfections were performed using the Lipofectamine ${ }^{\mathrm{TM}} 2000$ transfection reagent (Thermo Fisher Scientific Inc., Waltham, MA, USA).

\section{OGD-Treated Cardiomyocytes for In Vitro Study}

The cardiomyocytes were cultured in glucose-free Dulbecco's modified Eagle's medium (Thermo Fisher Scientific) in an anaerobic incubator $\left(1 \% \mathrm{O}_{2}, 5 \% \mathrm{CO}_{2}\right.$ and $94 \% \mathrm{~N}_{2}$ ) at $37{ }^{\circ} \mathrm{C}$ for $0 \mathrm{~h}, 6 \mathrm{~h}, 12 \mathrm{~h}, 18 \mathrm{~h}$, and $24 \mathrm{~h}$, respectively. The control cells were cultured under normoxic condition.

\section{Isolation and Identification of Exo}

Exo were extracted from the culture medium of different adMSCs using Total Exosome Isolation Reagent (\#4478359, Thermo Fisher Scientific) as previously reported [19]. The adMSC-specific medium was first ultra-centrifuged at $100,000 \times g$ overnight to exhaust the original exosomes in the medium, and the
adMSCs were cultured in the medium for $48 \mathrm{~h}$. After that, the culture medium was centrifuged at $2,000 \times \mathrm{g}$ for $30 \mathrm{~min}$ to discard cell debris. Then, the supernatant was loaded in a new tube and mixed with isolation reagent $(\mathrm{v}: \mathrm{v}=2: 1)$. The mixture was cultured at $4{ }^{\circ} \mathrm{C}$ overnight and then centrifuged at $10,000 \times g$ at $4{ }^{\circ} \mathrm{C}$ for $1 \mathrm{~h}$. The Exo spheres were resuspended in PBS for further use.

Then, the ultrastructure of the Exo was observed under a transmission electron microscope (TEM, Libra 120; Zeiss, Oberkochen, Germany). Distribution of particle size was analyzed by nanoparticle tracking analysis (NTA) using a Nanosight LM10 (Malvern Instruments, Malvern, UK) and the NTA v.3.0 software (Malvern Instruments). Expression of exosome biomarkers CD63 (ab217345, Abcam Inc., Cambridge, MA, USA) and CD81 (ab109201, Abcam) in the particles was examined by western blot analysis.

\section{Uptake of Exo by the Cardiomyocytes}

The extracted exosomes were labeled using a PKH26 red fluorescence kit (Sigma-Aldrich Chemical Company, St Louis, MO, USA) in accordance with the kit's instructions. Next, the Exo were resuspended and incubated with the mouse cardiomyocytes at $37{ }^{\circ} \mathrm{C}$ for $24 \mathrm{~h}$. After that, the cells were washed twice in PBS, fixed in $4 \%$ paraformaldehyde for $10 \mathrm{~min}$, and stained with 4', 6-diamidino-2-phenylindole. The PKH26labeled exosomes internalized by cardiomyocytes were observed under a confocal laser scanning microscope (Leica, Solms, Germany).

\section{3-(4, 5-Dimethylthiazol-2-yl)-2, 5-Diphenyltetrazolium Bromide (MTT) Assay}

Viability of cells was examined using an MTT kit (Beyotime Biotechnology Co. Ltd., Shanghai, China). In brief, the cells incubated in 96-well plates were incubated with MTT solution (10 $\mu \mathrm{L}$ per well) for $4 \mathrm{~h}$. Thereafter, each well was added with $100 \mu \mathrm{L}$ formazan solution to dissolve the formazan sediments. After 3-4 h, the violet formazan crystals were fully dissolved, and the optical density (OD) value at $570 \mathrm{~nm}$ was evaluated using a spectrophotometer to examine the viability of cells.

\section{Cell Apoptosis Detection by Flow Cytometry}

An Annexin V-fluorescein isothiocyanate (FITC) cell apoptosis detection kit (Thermo Fisher Scientific) was used to evaluate cell apoptosis according to the 
kit's instructions. In brief, the cells were incubated with $5 \mu \mathrm{L}$ propidium iodide and $5 \mu \mathrm{L}$ Annexin VFITC at room temperature in the dark for $5 \mathrm{~min}$. Thereafter, the apoptotic rate in cells was examined using a flow cytometer (FACS Verse, BD, Biosciences, Franklin Lakes, NJ, USA), and the data were analyzed by the Flow J software (Tree-star Inc, San Carlos, CA, USA).

\section{Reverse Transcription-Quantitative Polymerase Chain Reaction}

Total RNA from cells or tissues was extracted using RNAiso Plus (Takara Holdings Inc., Kyoto, Japan) and quantified using a NanoDrop spectrophotometer (Thermo Fisher Scientific). Next, 500 ng RNA was reversely transcribed into first-strand cDNA using an M-MLV Reverse Transcriptase (Invitrogen, Thermo Fisher Scientific). After that, real-time qPCR was conducted using the VeriQuest Fast SYBR Green qPCR Master Mix (Thermo Fisher Scientific) on a StepOneplus Real-time PCR System (Applied Biosystems, Carlsbad, CA, USA). The primer sequences are listed in Table 1, in which 5s and GAPDH were used for the internal loading for miRNA and mRNA, respectively. The fold change of gene expression was calculated by $2^{-\Delta \Delta \mathrm{CT}}$ method.

\section{Western Blot Analysis}

Total protein from the cardiomyocytes was extracted using radio-immunoprecipitation assay lysis buffer (Beyotime), and the protein concentration was assessed utilizing a bicinchoninic acid assay kit (Thermo Fisher Scientific). An equal volume of protein sample $(50 \mu \mathrm{g})$ was separated by $12 \%$ sodium dodecyl sulfatepolyacrylamide gel electrophoresis and transferred to polyvinylidene fluoride membranes. The membranes were blocked in $5 \%$ bovine serum albumin at $20^{\circ} \mathrm{C}$ for $2 \mathrm{~h}$ and incubated with the primary antibodies against B-cell lymphoma-2 (Bcl-2, 1:1,000, \#3498, Cell Signaling Technologies (CST), Beverly, MA, USA), Bcl-2-associated X (Bax, 1:1,000, \#2772, CST), $\alpha$-smooth muscle actin $(\alpha$ SMA, 1:1,000, \#19245, CST), TGFBR2 (1:1,000, ab269279, Abcam), Smad2 (1:1,000, ab63356, Abcam), p-Smad2 (phospho S255, 1:500, ab188334, Abcam), and $\operatorname{GAPDH}\left(1: 10,000\right.$, ab181603, Abcam) at $4{ }^{\circ} \mathrm{C}$ overnight. Further, the membranes were incubated with secondary antibodies against goat anti-mouse IgG H\&L (HRP) $(1: 10,000, a b 205719$, Abcam $)$ or goat anti-rabbit IgG H\&L (HRP) (1:10,000, ab205718, Abcam). The protein bands were developed using a BeyoECL Plus kit
(Beyotime). Relative gene expression was detected using the Image J software (version 1.8.0, NIH) with GAPDH as the internal loading protein.

\section{Enzyme-Linked Immunosorbent Assay}

The concentrations of inflammatory cytokines interleukin-6 (IL-6) and tumor necrosis factor- $\alpha$ (TNF- $\alpha$ ) in cardiomyocytes and tissue homogenate of mice were determined using a mouse IL-6 enzyme-linked immunosorbent assay (ELISA) kit (ab100713, Abcam) and a mouse TNF- $\alpha$ ELISA kit (H052, Jiancheng Bioengineering Institute, Nanjing, Jiangsu, China) according to the instructions of kits.

\section{MiRNA Microarray Analysis}

Differentially expressed miRNAs in cardiomyocytes after Exo treatment were screened using a miRNA microarray analysis performed by OE Biotech Company (Shanghai, China). Three samples from each group (OGD and Exo groups) were used. The samples were hybridized according the instructions of the Agilent-Mouse miRNA microarray $21.0(8-60 \mathrm{~K})$. The data from the raw microarray image were extracted using the Feature Extraction Software (Version 10.5, Agilent Technologies, Palo Alto, CA, USA) according the protocol of miRNA_105_Dec08 FE. After that, the data were visualized and analyzed using the GeneSpring GX Software (Version 10.0, Agilent).

\section{Dual-Luciferase Reporter Gene Assay}

The putative binding sequence between miR-671 and TGFBR2 3'UTR was first predicted on a bioinformatic system StarBase (http://starbase.sysu.edu.cn/ ). Then, the wild-type (WT) TGFBR2 3'UTR sequence containing the putative binding site with miR-671 and the corresponding mutant-type (MT) TGFBR2 sequence were cloned into the pmirGLO vector (Promega, Madison, WI, USA) to construct TGFBR2-WT and TGFBR2-MT luciferase reporter vectors. Thereafter, these reporters were coadministrated with miR-671 mimic or NC mimic into 293 T cells (ATCC, Manassas, VA, USA) and cultured for $48 \mathrm{~h}$. After that, the luciferase activity in cells was examined using a dual-luciferase reporter gene system (Promega).

\section{RNA-Binding Protein Immunoprecipitation Assay}

An RNA-binding protein immunoprecipitation (RIP) assay was performed according to the 
Table 1. Primer Sequences for RT-qPCR

\begin{tabular}{ll}
\hline Gene & Primer sequence (5'-3') \\
\hline miR-671 & F: GGAAGCCCTGGAGGGG \\
TGFBR2 & R: GAACATGTCTGCGTATCTC \\
NF2 & F: CCTACTCTGTCTGTGGATGACC \\
& R: GACATCCGTCTGCTTGAACGAC \\
FBXW11 & F: GCTCAGGACCTGGAGATGTATG \\
& R: CAGCCTGTTCTCAGGGTCATAG \\
DLG1 & F: TGCCTCCAGTATGATGAGCGAG \\
& R: GTCCATTGCTGAAGCGTAAGTGC \\
DVL3 & F: GTGAAAAGGCGGAAGCCAGCAT \\
& R: GTGCTGATTTCCAACACCTCCAG \\
YWHAZ & F: TGATGGACGCATTGAGCCAGGA \\
GAPDH & R: ACAATCTCCCGAAGGACTCGGA \\
5 s & F: CAGAAGACGGAAGGTGCTGAGA \\
& R: CTTTCTGGTTGCGAAGCATTGGG \\
\hline
\end{tabular}

$R T-q P C R$, reverse transcription quantitative polymerase chain reaction; miR-671, microRNA-671; TGBFR2, transforming growth factor beta (TGF- $\beta$ ) receptor 2; $N F-2$, neurofibromin 2; $F B X W 11$, F-box and WD repeat domain containing 11;DLG1, discs large MAGUK scaffold protein 1; DVL3, dishevelled segment polarity protein 3; YWHAZ, tyrosine 3-monooxygenase/tryptophan 5-monooxygenase activation protein zeta; GAPDH, glyceraldehyde-3-phosphate dehydrogenase; $F$, forward; $R$, reverse

instructions of an EZ-Magna RIP kit (Millipore Corp., Billerica, MA, USA). In brief, cardiomyocytes at an $80-90 \%$ confluence were lysed in RIP lysis buffer. Next, the cells lysates were co-incubated with magnetic beads conjugated with human anti-Ago2 (Millipore) or control anti-IgG (Millipore). After that, the samples were incubated with Proteinase $\mathrm{K}$ and the immunoprecipitated RNA was collected. Relative abundancy of miR-671 and TGFBR2 was then examined by RT-qPCR.

\section{Establishment of a Murine Model with MI}

Six- to eight-week-old C57BL/6JNifdc mice procured from Vital River Laboratory Animal Technology Co., Ltd. (Beijing, China) were housed in standard animal rooms in 12-12 $\mathrm{h}$ lights on-off cycle with free access to feed and water. After 1 week of acclimation, the mice were divided into following groups: sham group, MI group (model mice with MI), Exo-NC group (MI model mice were further injected with Exo-NC), and Exo-inhibitor group (MI model mice were further injected with Exoinhibitor), 8 in each group.

MI in mouse was induced as previously reported [20]. The animals were first anaesthetized through an intraperitoneal injection (i.p) of $100 \mathrm{mg} / \mathrm{kg}$ ketamine and $10 \mathrm{mg} / \mathrm{kg}$ xylazine. Then, MI in mouse was induced through a permanent ligation of the left anterior descending (LAD) artery using a 6-0 suture. Sham operation was performed in a similar manner except for permanent ligation. In the subsequent procedures, $100 \mu \mathrm{g}$ Exo-NC or Exo-inhibitor was dissolved in PBS and injected in the boundary area of the infarcted cardiac nearby the ligation site [21].

\section{Histological Examination}

Four weeks after MI induction, the animals were euthanized through an overdose of pentobarbital sodium $(150 \mathrm{mg} / \mathrm{kg}$, i.p). Then, the heart was crosscut, and a part of the myocardial tissues was used for the measurement of inflammatory cytokine production and gene expression, while the other part of tissues was used for histological examination.

The myocardial tissues were fixed in $4 \%$ paraformaldehyde at $4{ }^{\circ} \mathrm{C}$, dehydrated in serial ethanol, embedded in paraffin, and cut into sections. The fibrosis in tissues was examined using a Masson's trichrome staining kit (Solarbio Science \& Technology Co., Ltd., Beijing, China). Apoptosis rate of cardiomyocytes in tissues was examined using a Terminal deoxynucleotidyl transferase (TdT)-mediated dUTP nick end labeling (TUNEL) kit. In addition, the expression of TGFBR2 (1:50, ab61213, Abcam) and p-Smad2 (phospho S255, ab188334, Abcam) 
in the myocardial tissues nearby the infarcted area was examined by immunohistochemical (IHC) staining. After staining, the sections were observed under an Axiophot light microscope (Zeiss Inc, AG, Oberkochen, Germany) and photographed by the connected digital camera. Quantitative analysis was analyzed by the Image J software. The fibrosis rate was calculated according to the ratio of fibrotic area to the total area of the field of view, while the apoptosis rate was calculated according to the ratio of TUNELpositive cells to total cells in each field of view.

\section{Statistical Analysis}

GraphPad Prism 8 (GraphPad, La Jolla, CA, USA) was used for data analysis. Data were collected from three experiments and exhibited as mean \pm standard error of mean (SEM). Differences were analyzed by the unpaired $t$ test (two groups) or one-way or two-way analysis of variance (ANOVA) followed by Tukey's multiple test (multiple groups). $* p<0.05$ represents statistical significance.

\section{RESULTS}

\section{Identification of the adMSCs and the Exo}

First, the flow cytometry confirmed positive expression of the MSC surface biomarkers CD29, CD44, and CD99 while negative expression of CD45 in the acquired adMSCs (Fig. 1a). The subsequent alizarin red staining and Oil red $\mathrm{O}$ staining results confirmed the ability of the adMSCs in differentiating into osteoblasts and adipoblasts, respectively (Fig. 1b). These results suggested that the adMSCs were qualified for further use.

The particles extracted from adMSCs were identified as well. Under the TEM, the particles were observed in a typical oval shape (Fig. 1c). The NTA results suggested that the size of the extracted particles was mainly distributed in 50-120 nm (Fig. 1d). The subsequent western blot assay confirmed positive expression of exosome-specific biomarkers CD63 and CD81 in the extracted particles (Fig. 1e). Collectively, these results showed that the particles extracted from adMSCs were exosomes. Thereafter, to examine whether these Exo can be internalized by the cells, an exosome uptake assay was performed. After $24 \mathrm{~h}$ of incubation, it was found that the PHK26-labeled Exo were obviously internalized by the cardiomyocytes (Fig. 1f).

\section{Exo Treatment Protects Cardiomyocytes Against OGD-Induced Damage}

Mouse cardiomyocytes were treated with OGD to mimic an MI condition in vitro. The MTT assay suggested that the viability of cells was gradually weakened in the process of OGD treatment. After $12 \mathrm{~h}$, the viability of the cardiomyocytes reduced over a half (Fig. 2a). The apoptosis rate of the cardiomyocytes was examined by flow cytometry. As expected, the number of apoptotic cardiomyocytes was increased in the process of OGD treatment. After $12 \mathrm{~h}$, the apoptosis rate of cells was increased by two times compared to that at $0 \mathrm{~h}$ (Fig. 2b).

Next, the cardiomyocytes underwent $12 \mathrm{~h}$ of OGD treatment were then co-cultured with Exo. Those further treated with an equal volume of PBS. According to the MTT assay, the viability of cardiomyocytes was significantly suppressed by OGD treatment. Compared to PBS treatment, Exo treatment significantly restored the viability of cells (Fig. 2c). Also, the flow cytometry results showed that the OGD significantly enhanced the apoptosis of cardiomyocytes. Compared to PBS treatment, Exo treatment significantly reduced the apoptosis rate of cells (Fig. 2d).

Myocardial fibrosis and inflammation are key factors affecting MI prognosis [22, 23]. Here, we further examined the expression of apoptosis-related molecules Bax and Bcl-2 and the expression of the fibrosis biomarker $\alpha$-SMA in cells. The western blot analysis suggested that OGD treatment increased the expression of $\alpha$-SMA and the pro-apoptotic Bax while reduced the expression of anti-apoptotic Bcl-2. Importantly, the expression of Bax and $\alpha$-SMA was reduced while the expression of Bcl-2 was increased by Exo (Fig. 2e). The protein levels of IL- 6 and TNF- $\alpha$ in cells were examined using ELISA kits to evaluate the inflammatory response in cells. It was found that OGD led to a notable increase in the secretion of pro-inflammatory cytokines IL- 6 and TNF- $\alpha$ in the cardiomyocytes. Again, treatment of Exo reduced the secretion of IL-6 and TNF- $\alpha$ in cells (Fig. 2f). These results, collectively, confirmed the protective roles of adMSC-derived exosomes in OGD-treated cardiomyocytes.

\section{Exo Treatment Enhances miR-671 Expression in Cardiomyocytes}

As discussed above, exosomes frequently exert their functions through the delivery of miRNAs. Thereby, we examined the differentially expressed miRNAs in cardiomyocytes before and after Exo treatment using a 

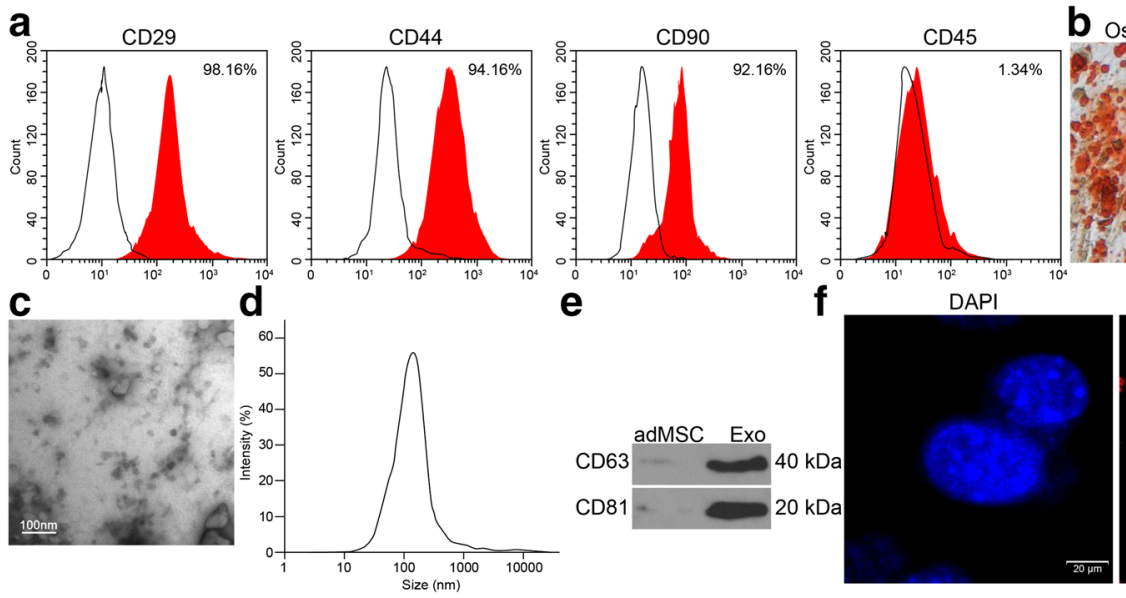

b Osteoblast differentiation
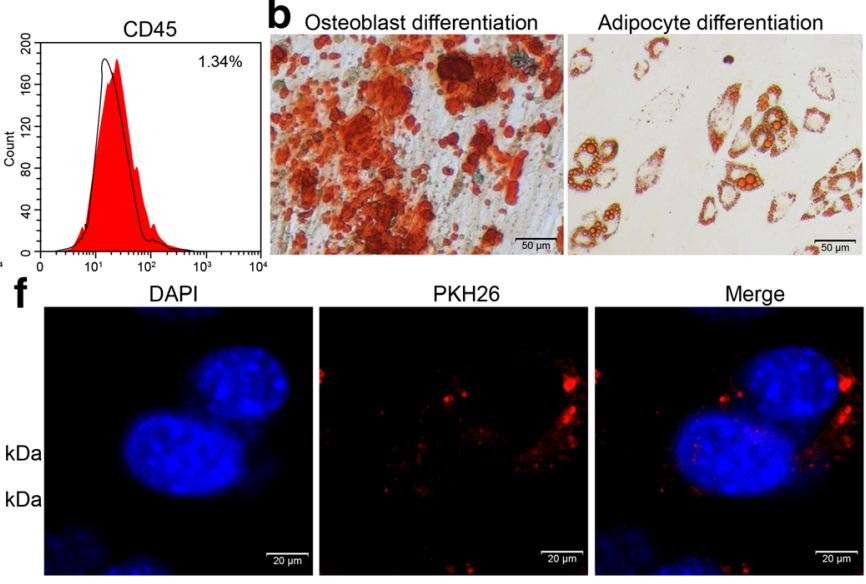

Fig. 1. Identification of the adMSCs and the Exo. a Expression of the MSC-surface positive marker proteins CD29, CD44, and CD99 and the negative marker protein CD45 in the acquired adMSCs examined by flow cytometry; b Osteogenic differentiation and adipogenic differentiation potentials of the adMSCs examined by alizarin red staining and Oil red O staining, respectively; $\mathbf{c}$ Morphology of the extracted particles observed under the TEM; d Particle size distribution examined by a NTA; $\mathbf{e}$ Expression of the exosome surface marker proteins determined by western blot analysis; $\mathbf{f}$ Uptake of exosomes by cardiomyocytes observed by the PHK26 labeling. Three independent experiments were performed. Representative images are presented.

miRNA microarray analysis. Using the fold change $\geq 2$ as the threshold, 10 mostly changed miRNAs in cardiomyocytes after Exo treatment are presented in Fig. $3 \mathrm{a}$, among which miR-671 showed the highest degree of upregulation (fold change $\approx 3$ ). Then, the RT-qPCR results showed that the miR-671 expression in cardiomyocytes was initially decreased after OGD treatment but then restored after further Exo treatment (Fig. 3b).
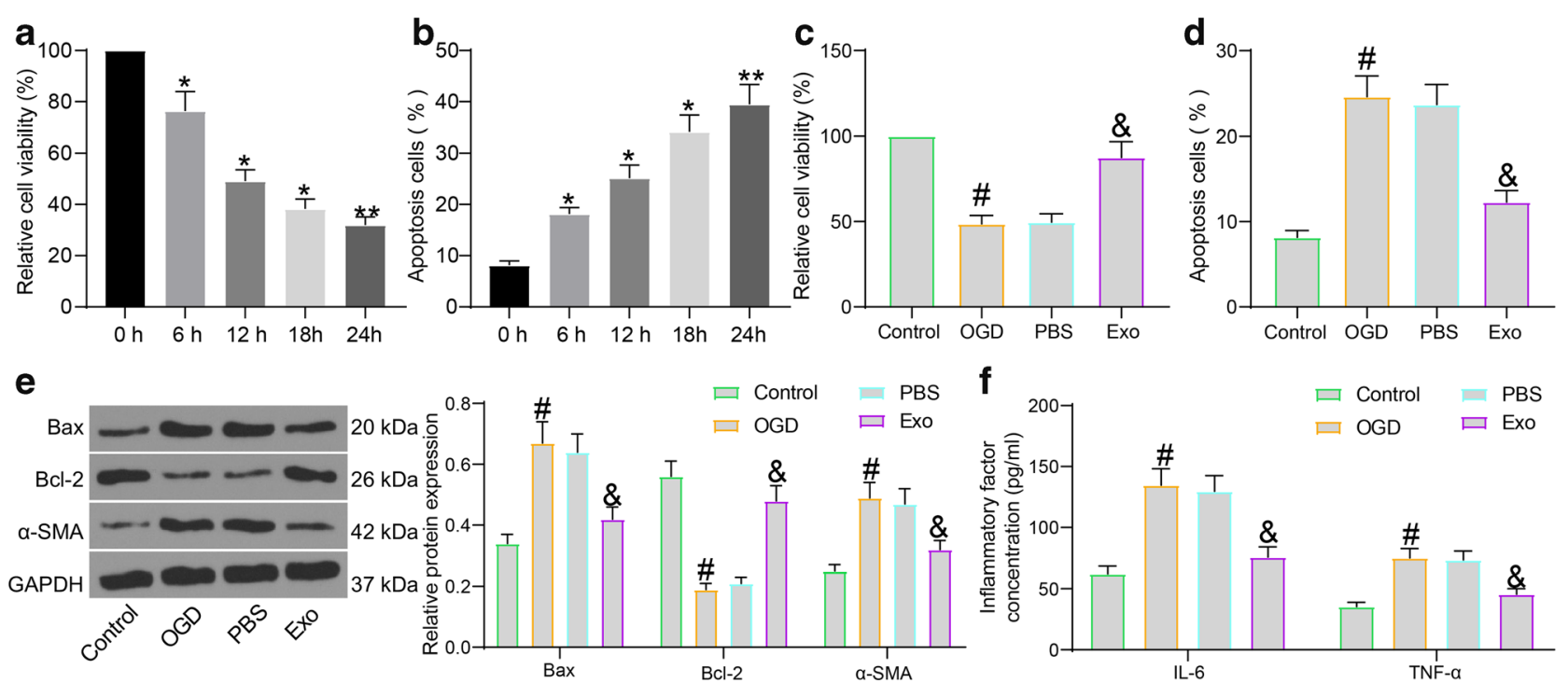

Fig. 2. Exo treatment protects cardiomyocytes against OGD-induced damage. a Viability of cardiomyocytes at different time points after OGD treatment determined by the MTT assay; b Apoptosis of cardiomyocytes at different time points after OGD treatment examined by flow cytometry; $\mathbf{c}$ Viability of cardiomyocytes after Exo treatment examined by the MTT assay; d Apoptosis of cardiomyocytes after Exo treatment detected by flow cytometry; e Protein levels of apoptosis-related factors (Bax and Bcl-2) and the fibrosis-related factor $\alpha$-SMA in OGD- and Exo-treated cardiomyocytes quantified by western blot analysis; f Production of pro-inflammatory cytokines (IL-6 and TNF- $\alpha$ ) in OGD- and Exo-treated cardiomyocytes determined using ELISA kits. Data were collected from three experiments and exhibited as mean \pm SEM. Differences were analyzed by one-way ANOVA (a-d) or two-way ANOVA (e, f); * $p<0.05$, $* * p<0.01$ vs. 0 h; \#p $<0.05$ vs. control group; $\& p<0.05$ vs. PBS group. 


\section{Downregulation of miR-671 Blocks the Protective Functions of Exo on Cardiomyocytes}

To validate whether miR-671 is responsible for the protective events mediated by the Exo, we first examined the abundancy of miR-671 in the exosome precipitates and the supernatant after centrifugation. Consequently, an enrichment of miR-671 was confirmed in the precipitated particles (Fig. 4a). Thereafter, interfering experiments were performed through administration of miR-671 inhibitor or the NC-inhibitor in the adMSCs, and the successful transfection was examined by RT-qPCR (Fig. 4b). Then, the exosomes extracted from these adMSCs were collected, named Exo-NC and Exo-inhibitor, correspondingly. Compared to Exo-NC, the Exo-inhibitor were found to have significantly reduced level of miR-671(Fig. 4c).

The OGD-treated cardiomyocytes were then treated with Exo-NC or Exo-inhibitor. In this setting, the MTT assay suggested that Exo-inhibitor reduced the viability of cardiomyocytes compared to Exo-NC (Fig. 4d). Then, the apoptosis rate of cells was examined by flow cytometry again. It was found that downregulation of miR-671 in Exo led to a notable increase in the number of apoptotic cardiomyocytes (Fig. 4e). The above results indicated that Exo treatment decreased the expression of $\alpha$-SMA and Bax while increased Bcl-2 expression in cells, while downregulation of miR-671 in Exo-inhibitor led to inverse trends (Fig. 4f). The ELISA results also found that the suppressive function of the Exo on the release of proinflammatory cytokines in cardiomyocytes was blocked upon miR-671 inhibition (Fig. 4g).

\section{miR-671 Directly Targets TGFBR2}

To identify the downstream molecules mediated by miR-671, we then predicted the possible target transcripts of miR-671 using several bioinformatic systems including TargetScan (http://www.targetscan.org/vert_72/), StarBase, miRwalk (http://mirwalk.umm.uniheidelberg.de/), miRDB (http://mirdb.org/), and miRDIP (http://ophid.utoronto.ca/mirDIP/), and 83 common outcomes were obtained (Fig. 5a). Then, a Kyoto Encyclopedia of Genes and Genomes (KEGG) enrichment analysis was performed based on these genes (Fig. 5b), and six key target genes including NF2, FBXW11, DLG, TGFBR2, DVL3, and YWHAZ were identified.

Then, miR-671 mimic and the NC mimic were transfected into the mouse cardiomyocytes, and then the expression of miR-671 and the mRNA expression of the above six genes was determined by RT-qPCR (Fig. 5c). It was found that miR-671 mimic enhanced the expression of miR-671 in cells and reduced the expression of TGFBR2. However, the expression of the reminding five candidate genes showed little change. Next, the putative binding site between miR-671 and TGFBR2 was obtained from

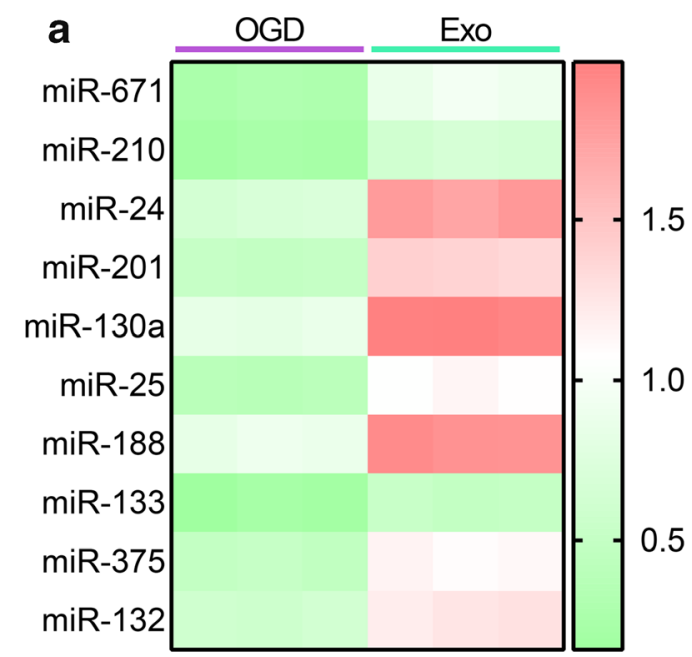

b

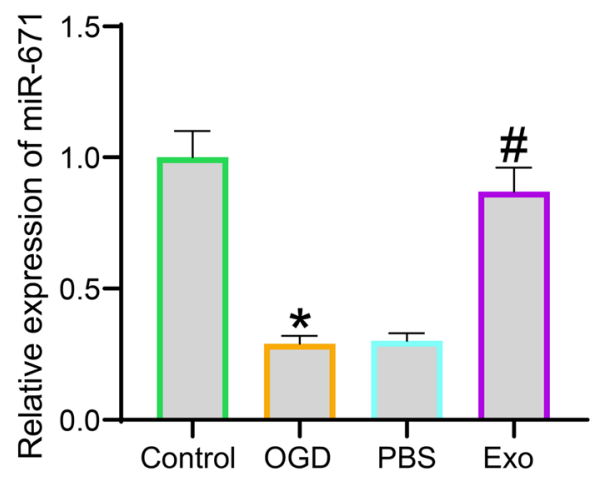

Fig. 3. Exo treatment enhances miR-671 expression in cardiomyocytes. a Top 10 differentially expressed miRNAs in cardiomyocytes before and after Exo treatment using a miRNA microarray analysis; b Expression of miR-671 in cardiomyocytes after OGD and Exo treatment determined by RT-qPCR. Data were collected from three experiments and exhibited as mean \pm SEM. Differences were analyzed by one-way ANOVA $(\mathbf{b}) ;{ }^{*} p<0.05$ vs. control group; $\# p<$ 0.05 vs. PBS group. 

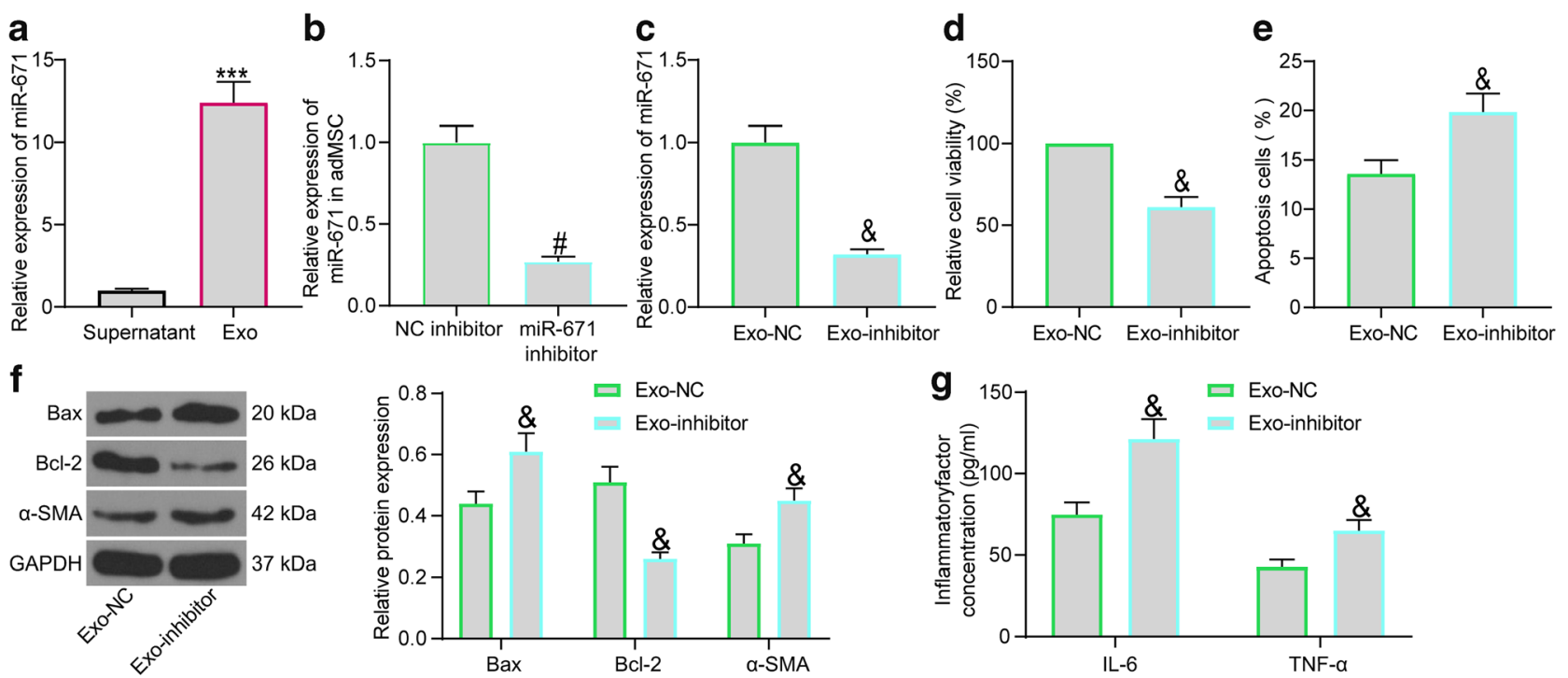

Fig. 4. Downregulation of miR-671 blocks the protective functions of Exo on cardiomyocytes. a miR-671 expression in the exosome precipitates and the supernatant after centrifugation using RT-qPCR; $\mathbf{b}$ Transfection efficiency of miR-671 inhibitor in adMSCs examined by RT-qPCR; $\mathbf{c}$ miR-671 expression in the exosomes determined by RT-qPCR; $\mathbf{d}$ Viability of OGD-cardiomyocytes after Exo-NC or Exo-inhibitor treatment determined by the MTT assay; $\mathbf{e}$ Apoptosis of OGD-cardiomyocytes after Exo-NC or Exo-inhibitor treatment examined by flow cytometry; $\mathbf{f}$ Protein levels of apoptosis-related factors (Bax and Bcl-2) and the fibrosis-related factor $\alpha$-SMA in OGD-cardiomyocytes after Exo-NC or Exo-inhibitor treatment determined by western blot analysis; $\mathbf{g}$ Production of pro-inflammatory cytokines (IL-6 and TNF- $\alpha$ ) in OGD-cardiomyocytes after Exo-NC or Exo-inhibitor treatment measured using ELISA kits. Data were collected from three experiments and exhibited as mean \pm SEM. Differences were analyzed by unpaired $t$ test (a-e) or two-way ANOVA (f, g); $* * * p<0.001$ vs. supernatant; $\# p<0.05$ vs. NC inhibitor group; $\& p<0.05$ vs. Exo-NC group.

StarBase to construct the TGFBR2-WT/TGFBR2-MT vectors (Fig. 5d). The vectors were co-transfected with miR-671 mimic or NC mimic into $293 \mathrm{~T}$ cells. The miR671 mimic was found to specifically suppressed the luciferase activity of TGFBR2-WT in cells (Fig. 5e). In addition, the subsequent RIP assay further confirmed that the miR-671 and TGFBR2 fragments were enriched by antiAgo2 compared to anti-IgG (Fig. 5f).

\section{Exosomal miR-671 Targets TGFBR2 and Reduces Smad2 Phosphorylation}

As mentioned before, TGFBR2 is a key regulator activating phosphorylation of Smad2. Activation of the Smad2 has been implicated in the pathogenesis of MI [24]. Here, the cardiomyocytes in the Exo-inhibitor group were further transfected with si-TGFBR2 or si-NC, and then the protein levels of TGFBR2, Smad2 and the phosphorylation of Smad2 in each group of cardiomyocytes were determined by western blot assays. It was found that OGD treatment significantly enhanced the level of TGFBR2 protein as well as the Smad2 activation in cells, while Exo-NC suppressed the protein level of TGFBR2 as well as the phosphorylation of Smad2. Again, these suppressive functions of Exo were diminished after miR671 knockdown. Further administration of si-TGFBR2 reduced the expression of TGFBR 2 and reduced phosphorylation of Smad2 in cells (Fig. 6a).

We then examined the viability of cardiomyocytes in the Exo-inhibitor group after further si-TGFBR2 or si-NC transfection. The MTT assay suggested that downregulation of TGFBR2 restored the viability of cardiomyocytes (Fig. 6b). As expected, the flow cytometry results showed that downregulation of TGFBR2 reduced the apoptosis rate of cardiomyocytes (Fig. 6c). The western blot assay suggested that downregulation of TGFBR2 suppressed the expression of Bax and $\alpha$-SMA and enhanced the expression of Bcl-2 in cardiomyocytes (Fig. 6d). In addition, the ELISA assay results suggested that si-TGFBR2 also suppressed the secretion of inflammatory cytokines including IL-6 and TNF- $\alpha$ in cells (Fig. 6e).

\section{Exosomal miR-671 Alleviates Fibrosis and Cell Apoptosis in Myocardial Tissues in Mice with MI}

A murine model of MI was induced, and the model mice were treated with Exo-NC or Exo-inhibitor. First, we 


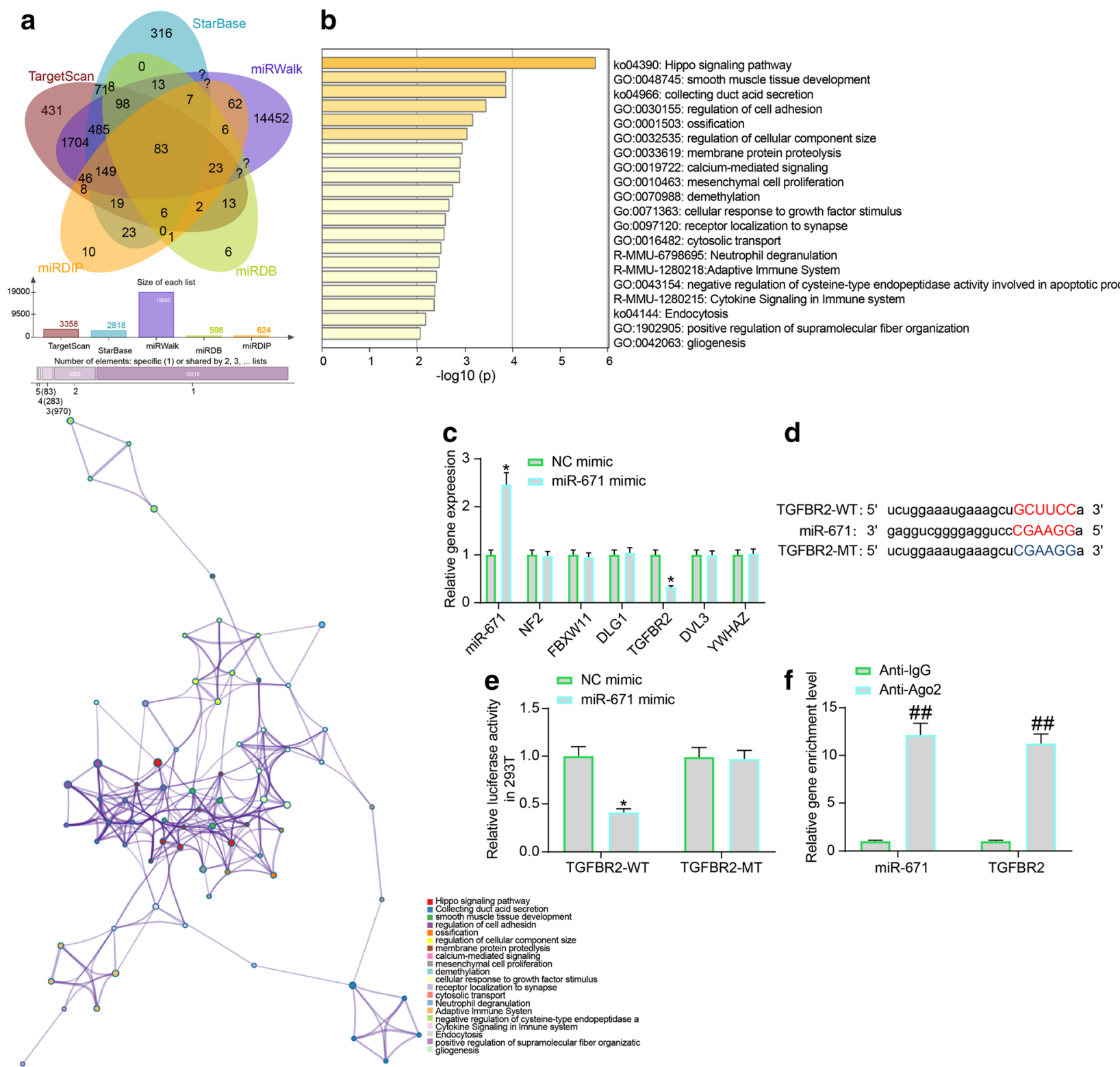

Fig. 5. miR-671 directly targets TGFBR2. a Putative target transcripts of miR-671 predicted using five bioinformatic systems; b A KEGG pathway enrichment analysis based on the above predicted mRNAs; $\mathbf{c}$ Expression of miR-671 and the mRNA expression of NF2, FBXW11, DLG, TGFBR2, DVL3, and YWHAZ in mouse cardiomyocytes after miR-671 mimic transfection examined by RT-qPCR; $\mathbf{d}$ Putative binding sequence between TGFBR2 and miR671 and the mutant binding sequence for the construction of luciferase vectors; e Binding relationship between TGFBR2 and miR-671 validated through a dual luciferase assay; $\mathbf{f}$ Enrichment of miR-671 and TGFBR2 fragments in the compounds pulled down by anti-Ago2 examined by the RIP assay. Data were collected from three experiments and exhibited as mean \pm SEM. Differences were analyzed by two-way ANOVA (c, e, and f); ${ }^{*} p<0.05 \mathrm{vs.} \mathrm{NC} \mathrm{mimic} \mathrm{group;}$ $\# \# p 0.01$ vs. anti-IgG.

examined the miR-671 expression in myocardial tissues nearby the infarcted area using RT-qPCR. Compared to the sham-operated ones, the mice in the MI group owned decreased expression of miR-671. Likewise, the expression of miR-671 was increased in mice after Exo-NC treatment but decreased after Exo-inhibitor treatment (Fig. 7a).

Then, the expression of TGFBR2 and p-Smad 2 in the murine myocardial tissues was examined by IHC staining. 


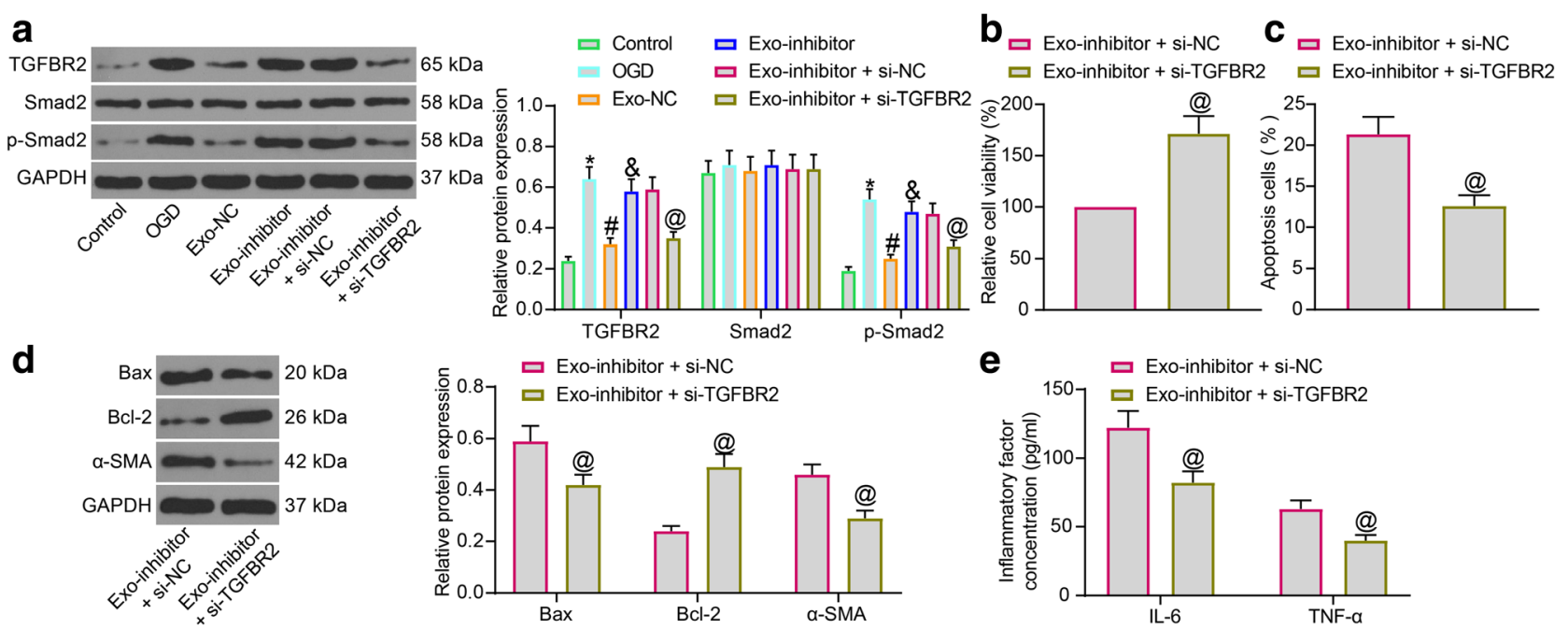

Fig. 6. Exosomal miR-671 targets TGFBR2 and reduces Smad2 phosphorylation. a Protein levels of TGFBR2, Smad2 and the phosphorylation of Smad2 in each group of cardiomyocytes examined by western blot analysis; $\mathbf{b}$ Viability of cardiomyocytes after si-TGFBR2 transfection measured by the MTT assay; $\mathbf{c}$ Apoptosis of cardiomyocytes after si-TGFBR2 transfection examined by flow cytometry; $\mathbf{d}$ Protein levels of apoptosis-related factors (Bax and Bcl-2) and the fibrosis-related factor $\alpha$-SMA in cardiomyocytes after si-TGFBR2 transfection determined by western blot analysis; e Production of pro-inflammatory cytokines (IL-6 and TNF- $\alpha$ ) in cardiomyocytes after si-TGFBR2 transfection determined using ELISA kits. Data were collected from three experiments and exhibited as mean \pm SEM. Differences were analyzed by unpaired $t$ test $(\mathbf{b}, \mathbf{c})$ or two-way ANOVA (a, d, and $\mathbf{e}) ;{ }^{*} p<0.05$ vs. Control group; \# $p<0.05$ vs. OGD group; $\& p<0.05$ vs. Exo-NC group; @ $p<0.05$ vs. Exo-inhibitor + si-NC group.

The staining intensity of TGFBR2 and p-Smad2 in the myocardial tissues in model mice was significantly enhanced. Further administration of Exo-NC reduced the protein levels of TGFBR2 and p-Smad2, while this reduction was blocked in the Exo-inhibitor group where miR671 expression was weakened (Fig. 7b). In addition, the concentrations of IL- 6 and TNF- $\alpha$ in the MI model mice were reduced by Exo-NC but elevated by Exo-inhibitor. Again, the inflammatory response in murine myocardial tissues was alleviated by Exo-NC, while the antiinflammatory function was significantly weakened in Exo-inhibitor (Fig. 7c).

Furthermore, Masson's trichrome staining suggested that Exo-NC reduced the myocardial fibrosis in the model mice with MI. However, the anti-fibrotic function of the exosomes was blocked again when miR-671 was suppressed (Fig. 7d). The TUNEL assay further confirmed increased cell apoptosis in the myocardial tissues in model mice. Treatment of Exo-NC alleviated cell apoptosis in vivo as well. Still, when the expression of miR-671 was inhibited, the anti-apoptotic effect of the Exo was notably weakened (Fig. 7e). These results indicated that miR-671 is at least partially accountable for the myocardial-protective roles of the Exo in vivo.

\section{DISCUSSION}

MI remains a major contributor to ischemic heart disease that represents a significant cause of morbidity and mortality across the global. Cellular therapy has seen promises and possibilities in the treatment for MI-induced ischemic heart disease, especially typical MSCs and their derived extracellular vesicles including exosomes [25]. In this study, we confirmed that Exo showed potent antiapoptotic, anti-inflammatory, and anti-fibrotic properties in vitro and in vivo through the delivery of miR-671 and the subsequent suppression of the TGFBR2/Smad2 axis.

The broad distribution of sources containing MSCs along the capacity of MSCs in differentiating into many mesenchymal phenotypes allows them as potential therapeutic candidates for multiple diseases including cardiac diseases and MI [26, 27]. MSC injection in the infarct area in a rat model with MI decreased the infarct size significantly [28]. The mediation of MSCs on the functions of surrounding cells such as cardiomyocyte, epithelial cells, and neurons has been reported as well [29, 30]. However, it is currently well-recognized that beneficial functions of MSCs are transient and achieved through the release of soluble paracrine factors and extracellular vesicles 

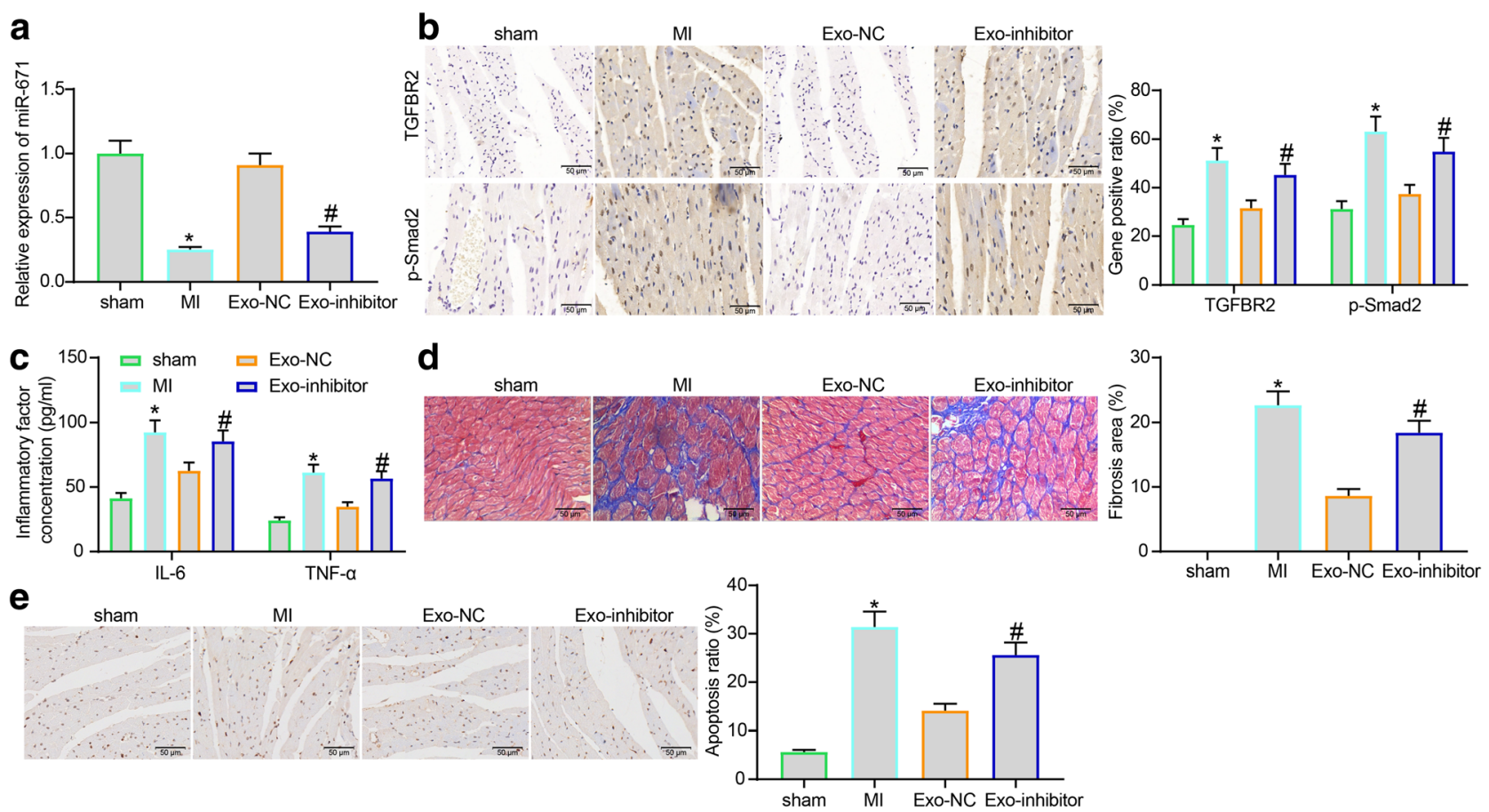

Fig. 7. Exosomal miR-671 alleviates fibrosis and cell apoptosis in myocardial tissues in mice with MI. a miR-671 expression in murine myocardial tissues nearby the infarcted area detected using RT-qPCR; b Protein levels of TGFBR2 and p-Smad2 in murine myocardial tissues nearby the infarcted area examined by IHC staining; c Concentrations of IL-6 and TNF- $\alpha$ in the homogenate of murine myocardial tissues examined by ELISA kits; $\mathbf{d}$ Myocardial fibrosis in mouse examined by masson's trichrome staining; e Cell apoptosis in murine myocardial tissues examined by TUNEL assay. $N=8$ in each group; representative images are provided. Data were collected from three experiments and exhibited as mean \pm SEM. Differences were analyzed by one-way ANOVA (a, d, and e) or two-way ANOVA (b, c); ${ }^{*} p<0.05$ vs. sham group; $\# p<0.05$ vs. Exo-NC group.

including exosomes [31-34]. Emerging evidence suggested the beneficial cardio-protective roles of MSCderived exosomes in reducing cell death and fibrosis [35, 36]. Here, we observed that Exo treatment enhanced the viability of OGD-treated cardiomyocytes and reduced the cell apoptosis rate as well. In a molecular perspective, the Exo treatment reduced the expression of pro-apoptotic Bax and fibrotic marker protein $\alpha$-SMA, but it elevated the expression of anti-apoptotic Bcl-2 in the OGD-treated cells. Reduced cell apoptosis, myocardial fibrosis, and inflammation were reproduced in vivo in a murine model with MI. These results collectively confirmed a cardioprotective role of the Exo.

MiRNAs are a major type of exosome cargos and are undoubtedly involved in almost every facet of repair mechanisms of MSC-based therapy in MI, including stem cell differentiation, apoptosis, neovascularization, cardiac remodeling, arrhythmias, and cardiac contractility and so forth [37]. For instance, localized injection of miRNA21-abundant extracellular vesicles enhanced cardiac function following MI [6]. Upregulation of miR-133 has been documented to enhance the therapeutic efficacy of MSCs on acute MI [38]. In the present research, a miRNA microarray analysis was performed to analyze the differentially expressed miRNAs in cardiomyocytes after Exo treatment, and miR-671 was identified as the miRNA with the highest degree of upregulation. Although its direct correlation with cardiac protection has been rarely concerned, miR-671 has been reported to be poorly expressed in patients with coronary artery disease [12]. In addition, exosomal miR671 has been suggested as a biomarker for the diagnosis of Kawasaki disease whose upregulation reduced vascular inflammation [39]. This miRNA has also shown antiinflammatory functions in osteoarthritis both in vitro and in vivo [40]. In order to confirm the involvement of miR671 in the Exo-mediated events, loss-of-function studies were performed by suppressing miR-671 expression in the adMSCs, and then the protective effects of the Exo were significantly blocked. Downregulation of miR-671 in exosomes increased cell apoptosis, inflammation, and myocardial fibrosis in OGD-treated cardiomyocytes and the model mice with MI. Therefore, we reported that miR- 


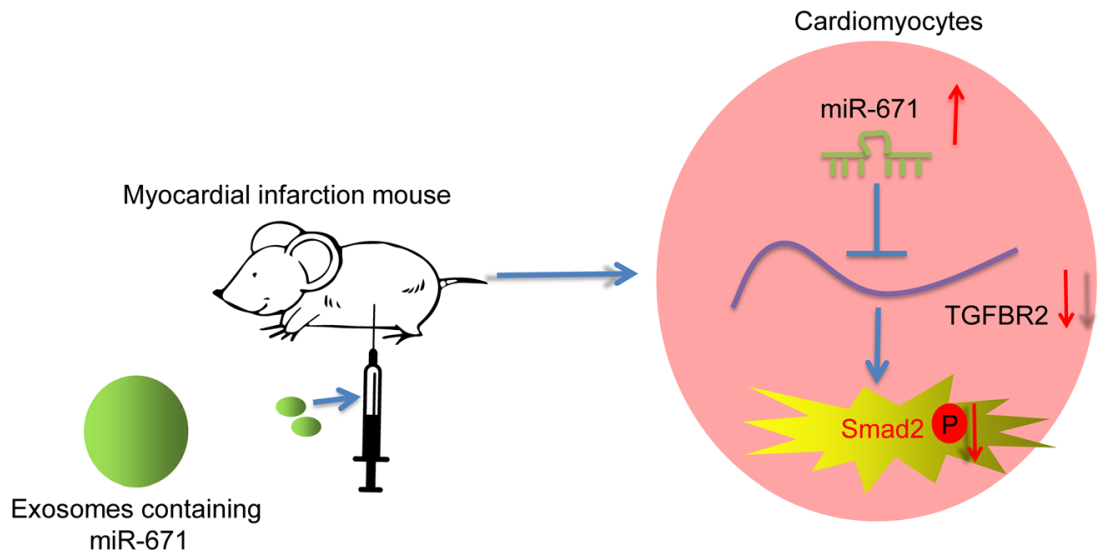

Fig. 8. A diagram for the molecular mechanism. AdMSC-derived exosomal miR-671 directly targets the TGFBR2/Smad2 axis, therefore alleviating OGDinduced damage on mouse cardiomyocytes in vitro and myocardial injury in model mice with MI.

671 has a cardio-protective role in MI and it is at least partially responsible for the events mediated by MSC and the exosomes.

It is reported that over $30 \%$ of protein-coding genes in human genome can be regulated by miRNAs, and they are inevitably implicated in the cascades mediated by miRNAs [41]. In our study, the integrated microarray analysis and cellular experiments suggested that miR-671 targets TGFBR2, an important receptor protein of the TGF- $\beta$ signaling pathway. As aforementioned, TGF- $\beta$ activates the TGFBRs to regulate the downstream Smad family. TGFBR2 has been reported as a positive regulator of Smad2 in several pathological conditions $[17,18,42,43]$. Also, the TGF- $\beta /$ Smad 2 pathway has been recognized as a traditional pathway which initiates activation of cardiac fibroblasts [44]. Targeting the TGF- $\beta / \mathrm{JNK}$ axis has also been validated to attenuate myocardial cell apoptosis in a rat model in the setting of MI [45]. Likewise, increased phosphorylation of Smad2 has been reported to be relevant to increased cardiomyocyte apoptosis, myocardial inflammation, and fibrosis [24, 46, 47]. Here, our study evidenced that the Exo reduced the expression of TGFBR2 as well as the Smad2 phosphorylation in OGD-treated cells. As expected, downregulation of miR-671 in exosomes reactivated the TGFBR2/Smad2 axis in cells. The additional loss-of-function assays found that downregulation of TFGBR2 also enhanced the viability while reduced apoptosis, fibrosis, and inflammation in cells. Similarly, a recent study by Yuan Lin et al. suggested that MSC-derived exosomes improved myocardial injury and fibrosis induced by diabetes mellitus through the inhibition of TGF- $\beta 1 /$ Smad2 signaling pathway [48].

\section{CONCLUSION}

To conclude, this study evidenced that adMSCderived exosomal miR-671 directly targets the TGFBR2/ Smad2 axis and alleviates OGD-induced damage on mouse cardiomyocytes in vitro and myocardial injury in model mice with MI (Fig. 8). This study validated the protective functions of Exo on myocardium and provided novel evidence for the possible clinical application of MSCs and the exosomes on MI management. We also hope more studies in this field will be conducted to provide more insights into the treatment of MI and heart diseases.

\section{AUTHOR CONTRIBUTION}

Xue Wang and Yuhai Zhu: Conceptualization, methodology, software; Chengcheng Wu: Data curation, statistical analysis; Wennan Liu and Yujie He: Visualization, experimental studies; Qing Yang: Manuscript preparation, reviewing and editing, validation; All authors read and approved the final manuscript.

\section{DATA AVAILABILITY}

The datasets used and/or analyzed during the current study are available from the corresponding author on reasonable request. 


\section{DECLARATIONS}

Ethics Approval. The research was ratified by the Animal Ethics Committee of Tianjin Medical University General Hospital. All animal procedures were performed in line with the Guide for the Care and Use of Laboratory Animals published by the National Institutes of Health (NIH, Bethesda, Maryland, USA). Great efforts were made to minimize the suffering of animals.

Consent for Publication. Not applicable.

Conflict of Interest. The authors declare no competing interests.

Open Access This article is licensed under a Creative Commons Attribution 4.0 International License, which permits use, sharing, adaptation, distribution and reproduction in any medium or format, as long as you give appropriate credit to the original author(s) and the source, provide a link to the Creative Commons licence, and indicate if changes were made. The images or other third party material in this article are included in the article's Creative Commons licence, unless indicated otherwise in a credit line to the material. If material is not included in the article's Creative Commons licence and your intended use is not permitted by statutory regulation or exceeds the permitted use, you will need to obtain permission directly from the copyright holder. To view a copy of this licence, visit http://creativecommons.org/licenses/by/4.0/.

\section{REFERENCES}

1. Buja, L.M., B. Zehr, L. Lelenwa, E. Ogechukwu, B. Zhao, A. Dasgupta, and R.F. Barth. 2020. Clinicopathological complexity in the application of the universal definition of myocardial infarction. Cardiovascular Pathology 44: 107153. https://doi.org/ 10.1016/j.carpath.2019.107153.

2. Thygesen, K., J.S. Alpert, A.S. Jaffe, B.R. Chaitman, J.J. Bax, D.A. Morrow, H.D. White, and Executive Group on behalf of the Joint European Society of Cardiology /American College of Cardiology /American Heart Association /World Heart Federation Task Force for the Universal Definition of Myocardial I. 2018. Fourth Universal Definition of Myocardial Infarction. Journal of the American College of Cardiology 72 (18): 2231-2264. https://doi.org/10.1016/ j.jacc.2018.08.1038.
3. Bejerano, T., S. Etzion, S. Elyagon, Y. Etzion, and S. Cohen. 2018. Nanoparticle delivery of miRNA-21 mimic to cardiac macrophages improves myocardial remodeling after myocardial infarction. Nano Letters 18 (9): 5885-5891. https://doi.org/10.1021/ acs.nanolett.8b02578.

4. Lippi, G., M. Franchini, and G. Cervellin. 2013. Diagnosis and management of ischemic heart disease. Seminars in Thrombosis and Hemostasis 39 (2): 202-213. https://doi.org/10.1055/s-00321333543.

5. Katarzyna, R. 2017. Adult stem cell therapy for cardiac repair in patients after acute myocardial infarction leading to ischemic heart failure: An overview of evidence from the recent clinical trials. Current Cardiology Reviews 13 (3): 223-231. https://doi.org/ 10.2174/1573403X13666170502103833.

6. Song, Y., C. Zhang, J. Zhang, Z. Jiao, N. Dong, G. Wang, Z. Wang, and L. Wang. 2019. Localized injection of miRNA-21-enriched extracellular vesicles effectively restores cardiac function after myocardial infarction. Theranostics 9 (8): 2346-2360. https://doi.org/ 10.7150/thno.29945.

7. Pan, W., Y. Zhu, X. Meng, C. Zhang, Y. Yang, and Y. Bei. 2019. Immunomodulation by exosomes in myocardial infarction. Journal of Cardiovascular Translational Research 12 (1): 28-36. https:// doi.org/10.1007/s12265-018-9836-7.

8. Chen, G.H., J. Xu, and Y.J. Yang. 2017. Exosomes: promising sacks for treating ischemic heart disease? American Journal of Physiology. Heart and Circulatory Physiology 313 (3): H508H523. https://doi.org/10.1152/ajpheart.00213.2017.

9. Hong, P., H. Yang, Y. Wu, K. Li, and Z. Tang. 2019. The functions and clinical application potential of exosomes derived from adipose mesenchymal stem cells: A comprehensive review. Stem Cell Research \& Therapy 10 (1): 242. https://doi.org/10.1186/s13287-0191358-y.

10. Yuan, M.J., T. Maghsoudi, and T. Wang. 2016. Exosomes mediate the intercellular communication after myocardial infarction. International Journal of Medical Sciences 13 (2): 113-116. https:// doi.org/10.7150/ijms.14112.

11. Sun, T., Y.H. Dong, W. Du, C.Y. Shi, K. Wang, M.A. Tariq, J.X. Wang, and P.F. Li. 2017. The role of microRNAs in myocardial infarction: From molecular mechanism to clinical application. International Journal of Molecular Sciences 18 (4). https://doi.org/ 10.3390/ijms18040745.

12. Zhong, Z., W. Zhong, Q. Zhang, Q. Zhang, Z. Yu, and H. Wu. 2020. Circulating microRNA expression profiling and bioinformatics analysis of patients with coronary artery disease by RNA sequencing. Journal of Clinical Laboratory Analysis 34 (1): e23020. https:// doi.org/10.1002/jcla.23020.

13. Shinde, A.V., and N.G. Frangogiannis. 2014. Fibroblasts in myocardial infarction: a role in inflammation and repair. Journal of Molecular and Cellular Cardiology 70: 74-82. https://doi.org/ 10.1016/j.yjmcc.2013.11.015.

14. Derynck, R., and Y.E. Zhang. 2003. Smad-dependent and Smadindependent pathways in TGF-beta family signalling. Nature 425 (6958): 577-584. https://doi.org/10.1038/nature02006.

15. Chen, Y., T. Li, Q. Gao, L.Y. Wang, and L.Q. Cui. 2018. MiR-1908 improves cardiac fibrosis after myocardial infarction by targeting TGF-beta1. European Review for Medical and Pharmacological Sciences 22 (7): 2061-2069. https://doi.org/10.26355/ eurrev_201804_14736.

16. Wang, L., H. Shi, J.L. Huang, S. Xu, and P.P. Liu. 2020. Linggui Zhugan Decoction () inhibits ventricular remodeling after acute 
myocardial infarction in mice by suppressing TGF-beta1/Smad signaling pathway. Chinese Journal of Integrative Medicine 26 (5): 345-352. https://doi.org/10.1007/s11655-018-3024-0.

17. Shi, X., Q. Liu, N. Li, W. Tu, R. Luo, X. Mei, Y. Ma, W. Xu, H. Chu, S. Jiang, Z. Du, H. Zhao, L. Zhao, L. Jin, W. Wu, and J. Wang. 2018. MiR-3606-3p inhibits systemic sclerosis through targeting TGF-beta type II receptor. Cell Cycle 17 (16): 1967-1978. https:// doi.org/10.1080/15384101.2018.1509621.

18. Zhu, X., T. Zhang, Y. Zhang, H. Chen, J. Shen, X. Jin, J. Wei, E. Zhang, M. Xiao, Y. Fan, R. Mao, and G. Zhou. 2020. A superenhancer controls TGF- beta signaling in pancreatic cancer through downregulation of TGFBR2. Cellular Signalling 66: 109470. https://doi.org/10.1016/j.cellsig.2019.109470.

19. Peng, Y., J.L. Zhao, Z.Y. Peng, W.F. Xu, and G.L. Yu. 2020. Exosomal miR-25-3p from mesenchymal stem cells alleviates myocardial infarction by targeting pro-apoptotic proteins and EZH2. Cell Death \& Disease 11 (5): 317. https://doi.org/10.1038/s41419-0202545-6.

20. Li, X., X. Xue, Y. Sun, L. Chen, T. Zhao, W. Yang, Y. Chen, and Z. Zhang. 2019. MicroRNA-326-5p enhances therapeutic potential of endothelial progenitor cells for myocardial infarction. Stem Cell Research \& Therapy 10 (1): 323. https://doi.org/10.1186/s13287019-1413-8.

21. Sun, L., W. Zhu, P. Zhao, Q. Wang, B. Fan, Y. Zhu, Y. Lu, Q. Chen, J. Zhang, and F. Zhang. 2020. Long noncoding RNA UCA1 from hypoxia-conditioned hMSC-derived exosomes: A novel molecular target for cardioprotection through miR-873-5p/XIAP axis. Cell Death \& Disease 11 (8): 696. https://doi.org/10.1038/s41419-02002783-5.

22. Bai, W.W., Z.Y. Tang, T.C. Shan, X.J. Jing, P. Li, W.D. Qin, P. Song, B. Wang, J. Xu, Z. Liu, H.Y. Yu, Z.M. Ma, S.X. Wang, C. Liu, and T. Guo. 2020. Up-regulation of paired-related homeobox 2 promotes cardiac fibrosis in mice following myocardial infarction by targeting of Wnt5a. Journal of Cellular and Molecular Medicine 24 (3): 2319-2329. https://doi.org/10.1111/jcmm.14914.

23. Eid, R.A., M.A. Khalil, M.A. Alkhateeb, S.M. Eleawa, M.S.A. Zaki, A.F. El-Kott, M. Al-Shraim, F. El-Sayed, M.A. Eldeen, M.M. BinMeferij, K.M.E. Awaji, and A.S. Shatoor. 2020. Exendin-4 attenuates remodeling in the remote myocardium of rats after an acute myocardial infarction by activating beta-arrestin- 2 , protein phosphatase $2 \mathrm{a}$, and glycogen synthase kinase- 3 and inhibiting beta-catenin. Cardiovascular Drugs and Therapy. https://doi.org/10.1007/ s10557-020-07006-9.

24. Xu, H.M., F.H. Sui, M.H. Sun, and G.L. Guo. 2019. Downregulated microRNA-224 aggravates vulnerable atherosclerotic plaques and vascular remodeling in acute coronary syndrome through activation of the TGF-beta/Smad pathway. Journal of Cellular Physiology 234 (3): 2537-2551. https://doi.org/10.1002/jcp.26945.

25. Peng, H., and A. Abdel-Latif. 2019. Cellular therapy for ischemic heart disease: An update. Advances in Experimental Medicine and Biology 1201: 195-213. https://doi.org/10.1007/978-3-030-31206-0_10.

26. Shafei, A.E., M.A. Ali, H.G. Ghanem, A.I. Shehata, A.A. Abdelgawad, H.R. Handal, K.A. Talaat, A.E. Ashaal, and A.S. ElShal. 2017. Mesenchymal stem cell therapy: A promising cell-based therapy for treatment of myocardial infarction. The Journal of Gene Medicine 19 (12). https://doi.org/10.1002/jgm.2995.

27. Zhang, F.B., and H.T. Yang. 2006. Plasticity of bone marrow mesenchymal stem cells differentiating into cardiomyocytes and the potential of cardiac therapeutics. Sheng Li Ke Xue Jin Zhan 37 (3): 199-204.

28. Yoon, J., B.G. Min, Y.H. Kim, W.J. Shim, Y.M. Ro, and D.S. Lim. 2005. Differentiation, engraftment and functional effects of pretreated mesenchymal stem cells in a rat myocardial infarct model.
Acta Cardiologica 60 (3): 277-284. https://doi.org/10.2143/ AC.60.3.2005005.

29. Jones, S., N. Horwood, A. Cope, and F. Dazzi. 2007. The antiproliferative effect of mesenchymal stem cells is a fundamental property shared by all stromal cells. Journal of Immunology 179 (5): 2824 2831. https://doi.org/10.4049/jimmunol.179.5.2824.

30. Muguruma, Y., T. Yahata, H. Miyatake, T. Sato, T. Uno, J. Itoh, S. Kato, M. Ito, T. Hotta, and K. Ando. 2006. Reconstitution of the functional human hematopoietic microenvironment derived from human mesenchymal stem cells in the murine bone marrow compartment. Blood 107 (5): 1878-1887. https://doi.org/10.1182/blood2005-06-2211.

31. Biancone, L., S. Bruno, M.C. Deregibus, C. Tetta, and G. Camussi. 2012. Therapeutic potential of mesenchymal stem cell-derived microvesicles. Nephrology, Dialysis, Transplantation 27 (8): 3037-3042. https://doi.org/10.1093/ndt/gfs168.

32. Borger, V., M. Bremer, R. Ferrer-Tur, L. Gockeln, O. Stambouli, A. Becic, and B. Giebel. 2017. Mesenchymal stem/stromal cell-derived extracellular vesicles and their potential as novel immunomodulatory therapeutic agents. International Journal of Molecular Sciences 18 (7). https://doi.org/10.3390/ijms18071450.

33. Ju, C., Y. Shen, G. Ma, Y. Liu, J. Cai, I.M. Kim, N.L. Weintraub, N. Liu, and Y. Tang. 2018. Transplantation of cardiac mesenchymal stem cell-derived exosomes promotes repair in ischemic myocardium. Journal of Cardiovascular Translational Research 11 (5): 420 428. https://doi.org/10.1007/s12265-018-9822-0.

34. Lai, R.C., F. Arslan, M.M. Lee, N.S. Sze, A. Choo, T.S. Chen, M. Salto-Tellez, L. Timmers, C.N. Lee, R.M. El Oakley, G. Pasterkamp, D.P. de Kleijn, and S.K. Lim. 2010. Exosome secreted by MSC reduces myocardial ischemia/reperfusion injury. Stem Cell Research 4 (3): 214-222. https://doi.org/10.1016/j.scr.2009.12.003.

35. Shi, Y., Y. Yang, Q. Guo, Q. Gao, Y. Ding, H. Wang, W. Xu, B. Yu, M. Wang, Y. Zhao, and W. Zhu. 2019. Exosomes derived from human umbilical cord mesenchymal stem cells promote fibroblastto-myofibroblast differentiation in inflammatory environments and benefit cardioprotective effects. Stem Cells and Development 28 (12): 799-811. https://doi.org/10.1089/scd.2018.0242.

36. Zhu, L.P., T. Tian, J.Y. Wang, J.N. He, T. Chen, M. Pan, L. Xu, H.X Zhang, X.T. Qiu, C.C. Li, K.K. Wang, H. Shen, G.G. Zhang, and Y.P. Bai. 2018. Hypoxia-elicited mesenchymal stem cell-derived exosomes facilitates cardiac repair through miR-125b-mediated prevention of cell death in myocardial infarction. Theranostics 8 (22): 6163-6177. https:// doi.org/10.7150/thno. 28021

37. Wen, Z., S. Zheng, C. Zhou, W. Yuan, J. Wang, and T. Wang. 2012. Bone marrow mesenchymal stem cells for post-myocardial infarction cardiac repair: MicroRNAs as novel regulators. Journal of Cellular and Molecular Medicine 16 (4): 657-671. https://doi.org/ 10.1111/j.1582-4934.2011.01471.x.

38. Chen, Y., Y. Zhao, W. Chen, L. Xie, Z.A. Zhao, J. Yang, Y. Chen, W. Lei, and Z. Shen. 2017. MicroRNA-133 overexpression promotes the therapeutic efficacy of mesenchymal stem cells on acute myocardial infarction. Stem Cell Research \& Therapy 8 (1): 268. https://doi.org/10.1186/s13287-017-0722-z.

39. Zhang, X., G. Xin, and D. Sun. 2018. Serum exosomal miR-328, miR-575, miR-134 and miR-671-5p as potential biomarkers for the diagnosis of Kawasaki disease and the prediction of therapeutic outcomes of intravenous immunoglobulin therapy. Experimental and Therapeutic Medicine 16 (3): 2420-2432. https://doi.org/ 10.3892/etm.2018.6458.

40. Zhang, B., M. Sun, J. Wang, C. Ma, T. Hao, G. Liu, G. Bao, and Y. Zhu. 2019. MiR-671 ameliorates the progression of osteoarthritis in vitro and in vivo. Pathology, Research and Practice 215 (7): 152423. https:// doi.org/10.1016/j.prp.2019.04.015. 
41. Lakshmipathy, U., and R.P. Hart. 2008. Concise review: MicroRNA expression in multipotent mesenchymal stromal cells. Stem Cells 26 (2): 356-363. https://doi.org/10.1634/stemcells.2007-0625.

42. Liu, S., J. Iaria, R.J. Simpson, and H.J. Zhu. 2018. Ras enhances TGF-beta signaling by decreasing cellular protein levels of its type II receptor negative regulator SPSB1. Cell Communication and Signaling: CCS 16 (1): 10. https://doi.org/10.1186/s12964-018-0223-4.

43. Pan, L.X., and W. Ding. 2020. LncRNA HAGLR accelerates femoral neck fracture healing through negatively regulating miRNA19a-3p. European Review for Medical and Pharmacological Sciences 24 (8): 4080-4087. https://doi.org/10.26355/ eurrev_202004_20984.

44. Chen, P., R. Wu, W. Zhu, Z. Jiang, Y. Xu, H. Chen, Z. Zhang, H. Chen, L. Zhang, H. Yu, J. Wang, and X. Hu. 2014. Hypoxia preconditioned mesenchymal stem cells prevent cardiac fibroblast activation and collagen production via leptin. PLOS ONE 9 (8): e103587. https://doi.org/10.1371/journal.pone.0103587.

45. Zhou, F.Q., X.F. Zhao, F.Y. Liu, S.S. Wang, H.L. Hu, and Y. Fang. 2019. MiR-101a attenuates myocardial cell apoptosis in rats with acute myocardial infarction via targeting TGF-beta/JNK signaling pathway. European Review for Medical and Pharmacological Sciences 23 (10): 4432-4438. https://doi.org/10.26355/ eurrev_201905_17952.

46. Ding, Y.F., Y.R. Peng, J. Li, H. Shen, M.Q. Shen, and T.H. Fang. 2013. Gualou Xiebai Decoction prevents myocardial fibrosis by blocking TGF-beta/Smad signalling. The Journal of Pharmacy and Pharmacology 65 (9): 1373-1381. https://doi.org/10.1111/ jphp. 12102.

47. He, Y., X. Zhou, X. Zheng, and X. Jiang. 2013. Exogenous highmobility group box 1 protein prevents postinfarction adverse myocardial remodeling through TGF-beta/Smad signaling pathway. Journal of Cellular Biochemistry 114 (7): 1634-1641. https:// doi.org/10.1002/jcb.24505.

48. Lin, Y., F. Zhang, X.F. Lian, W.Q. Peng, and C.Y. Yin. 2019. Mesenchymal stem cell-derived exosomes improve diabetes mellitus-induced myocardial injury and fibrosis via inhibition of TGF-beta1/Smad2 signaling pathway. Cellular and Molecular Biology (Noisy-le-Grand, France) 65 (7): 123-126. 